\title{
Análise do modelo de atenção à saúde bucal em municípios do estado de Pernambuco
}

\author{
Analysis of the oral health care model \\ in cities of the state of Pernambuco
}

Petrônio J osé de Lima M artelli ${ }^{1}$

A manda Priscila de Santana Cabral ${ }^{1}$

Fernando Castim Pimentel ${ }^{1}$

Cícera Lissandra Sá Vieira M acedo ${ }^{1}$

I ve da Silva M onteiro ${ }^{1}$

Shirley Florêncio da Silva ${ }^{1}$

${ }^{1}$ Centro de Pesquisas Aggeu M agalhães, Fundação Oswaldo Cruz. Av.

Professor M oraes Rego, s/n, Cidade Universitária. 50670-420 Recife PE. nandocastim@yahoo.com.br
Abstract The objective of this study was to analyze the oral health care model in nine cities of thestate of Pernambuco on the basis of statements of the Oral Health Primary Care Coordinators. Semi-structured interviews were conducted with the coordinators for collecting data about theoral health care model adopted by the local authorities, about their activities as coordinators, their qualification, the practices developed by the dental surgeon making part of the Family $\mathrm{H}$ ealth Team, and about basic care procedures and reference and counter-reference networks. The data collected in the interviews were evaluated using a qualitative methodology and a matrix was created for classifying the cities according to the profile of their professionals and the actions and services offered. The results showed that among the analyzed cities three fitted into the category "structured", five into the category "semi-structured" and only one was considered "not-structured". This indicates that the greater of the cities is facing difficulties in structuring oral health care, mainly as refers to incorporation of new care practices and to the professional qualification of dental surgeons and oral health coordinators. Key words Oral health, Family H ealth Program, $\mathrm{H}$ ealth policy
Resumo 0 presente estudo teve como objetivo analisar o modelo de atenção à saúde bucal em nove municípios do estado de Pernambuco, a partir do relato dos seus coordenadores de saúde bucal. Realizou-se uma entrevista semi-estruturada com cada um dos coordenadores sobreo modelo de saúdebucal adotado pelo município, suas atividades como coordenador, formação e práticas desenvolvidas pelo cirurgião-dentista inserido na EquipedeSaúde da Família, procedi mentos de aten ção básica e rede de referência e contra-referência. 0 estudo utilizou uma metodologia qualitativa para avaliar os dados obtidos nas entrevistas e foi criada uma matriz dequalificação dos municípios, em que os mesmos foram classificados, de acordo com o perfil dos profissionais e das ações e serviços oferecidos pelo município. Os resultados mostraram que dos municípios analisados, três enquadram-se na categoria "estruturado", cinco na categoria "semi-estruturado" e apenas um como "não-estruturado", demonstrando quea maioria dos municípios apresenta dificuldades na estruturação da saúde bucal, principalmente nas questões relacionadas à incorporação de novas práticas assistenciaiseà qualificação profissional dos cirurgiões-dentistas e dos coordenadores de saúde bucal.

Palavras-chave Saúdebucal, Programa Saúdeda Família, Política de saúde 
Introdução

A incorporação da saúde bucal à Equipe de Saúdeda Família (ESF) ocorreu no final do ano 2000, quando o M inistério da Saúde (M S) normatizou e passou a incentivar financeiramente a constituição de Equipes de Saúde Bucal (ESB) ${ }^{1}$, criando-se a possibilidade de se instituir um novo paradigma de planejamento e programação da atenção básica, representando a mais importante iniciativa de assistência pública, expandindo e reorganizando as atividades de saúde bucal de acordo com os princípios e diretrizes do SU S2.

Oliveira et al. ${ }^{3}$ destacaram que as experiências de introdução da saúde bucal naESF não ape nas transferiram linearmente 0 espaço de trabaIho do cirurgião-dentista (CD) para além do consultório, mas produziram um ganho no sentido de reordenamento da prática odontológica.

Em Pernambuco, percebe-se uma evolução quantitativa das Equipes de Saúde Bucal, passando de 237 em 2002 para 721 equipes implantadas em 20054. Entretanto, também éfundamental para o sucesso do modelo o aumento da cobertura odontológica, uma melhor qualificação profissional, uma rede de referência e contra-referência estruturada, além da participação da comunidade, o que tornaria os municípios mais adequados a desenvolver a atenção à saúde bucal de forma ampla, conforme os princípios do SUS.

A escolha do Programa Saúde da Família (PSF) como estratégia de reorientação do modelo assistencial vigentedesencadeia mudanças profundas deordem conceitual, metodológica eope racional na prática profissional dos envolvidos. Com isso, a tônica desse processo tem sido dada a partir de novos papéis sociais e profissionais, nova organização do processo de trabalho e novos conhecimentos, proporcionando o desenvolvimento deações humanizadas, intersetorialmente articuladas e socialmente apropriadas ${ }^{5,6}$.

No campo da Atenção à Saúde, todos os profissionais devem estar aptos a desenvolver ações de promoção, prevenção, proteção e reabilitação em nível individual e coletivo; assegurar que sua prática seja realizada deforma integrada econtínua com as demais instâncias do sistema de saúde; realizar seus serviços dentro dos mais altos padrões de qualidade e dos princípios da bioética (ética da vida); ter em conta que a responsabilidade da atenção à saúde somente se encerra com a resolução do problema de saúde, tanto ao nível individual quanto coletivo ${ }^{7}$.

No setor saúde, a falência do perfil profissional e a inadequação às novas modalidades de or- ganização e prestação de serviços nunca foi tão visível. A formação de profissionais capazes de responder às necessidades do sistema, às de saúde da família e tantas outras é urgente nesse setor ${ }^{8}$.

Assim, o estudo do perfil dos CDs inseridos nas ESBs ea atuação dos Coordenadores de Saúde Bucal (CSB) têm um importante papel na estratégia de reorganização do modelo de atenção à saúde, pois esses profissionais têm a tarefa de compreender as realidades social, cultural e econômica do seu meio e direcionar suas ações para mudar esses aspectos em benefício da população. Diante disso, o presente trabal ho teve como objetivo analisar o perfil do CD e do CSB inseridos na ESF, assim como as características do modelo de atenção em saúde bucal em nove municípios do estado dePernambuco, sob o ponto de vista dos CSB.

\section{M etodologia}

0 presente estudo originou-se como parte do projeto de pesquisa intitulado "M odelo de atenção em saúdebucal eformação do cirurgião dentista na Estratégia de Saúde da Família do estado dePernambuco", desenvolvido no Centro dePesquisas Aggeu M agalhães (CPqAM), em parceria com a Associação Caruaruense de Ensino Superior e financiado pela Fundação de Amparo à Ciência e Tecnologia do Estado de Pernambuco (FACEPE). Consiste em um estudo descritivo com abordagem qualitativa, analisando a possível mudança da prática hegemônica do $C D$, com a sua incorporação na ESF, além de verificar que características essa estratégia vem adquirindo após a sua incorporação.

Para a amostra, foram tipificados, com intencionalidade, dez municípios, levando-seem conta critérios primários (tamanho populacional do município, possuir ESB) e critérios secundários (condição de gestão, sede de GERES, sede de M ódulo, possuir CEO, critérios regionais, cobertura da atenção básica em saúde bucal). Entretanto, participaram da amostra apenas os CSB de nove municípios, sendo estes: Arcoverde, Caetés, Caruaru, Chã Grande, Palmares, Petrolina, Recife, São José da Coroa Grande e São José do Egito.

0 objetivo do estudo foi exposto a todos os envolvidos queassinaram um termo deconsentimento livree esclarecido. Os dados foram coletados por intermédio de uma entrevista semi-estruturada com o CSB, gravada e transcrita pormenorizadamente após sua autorização, e referiam-se ao modelo deatenção em saúde bucal ado- 
tado pelo município, às suas atividades como CSB, à formação dos CDs que trabalham na atenção básica, à mudança na prática dos CDs inseridos na ESF, ao perfil da clientela atendida, à oferta de serviços eà rede de referência econtra-referência.

Para a análise dos dados, criou-se uma escala de mensuração, ou seja, questões de interesse deste trabalho foram elaboradas, formando um conjunto de categorias. Em seguida, os trechos mais relevantes de cada entrevista foram "recortados" e agrupados em cada uma das categorias, obedecendo a alguns critérios e recebendo uma pontuação específica (Quadro 1).

Quadro 1. Critérios para categorização dos municípios.

\begin{tabular}{|c|c|c|c|c|}
\hline Categorias & \multicolumn{4}{|c|}{ Critérios e pontuação } \\
\hline $\begin{array}{l}\text { Qualificação dos CDs } \\
\text { (Especialização em } \\
\text { Saúde Pública / Saúde } \\
\text { Coletiva) }\end{array}$ & $\begin{array}{l}\text { Nenhum CD } \\
\text { qualificado }(1)^{*}\end{array}$ & $\begin{array}{l}\text { Poucos CDs } \\
\text { qualificados }(2)^{*}\end{array}$ & $\begin{array}{l}\text { A maioria dos CDs } \\
\text { qualificados(3)* }\end{array}$ & $\begin{array}{l}\text { Todos os CDs } \\
\text { qualificados }(4)^{*}\end{array}$ \\
\hline $\begin{array}{l}\text { Mudança no perfil da } \\
\text { assistência após } \\
\text { incorporação do CD à } \\
\text { equipe }\end{array}$ & $\begin{array}{l}\text { Não houve mudança } \\
\text { no perfil da assistência } \\
(1)^{*}\end{array}$ & $\begin{array}{l}\text { Não, mas observou-se } \\
\text { a intencionalidade } \\
\text { para mudança }(2)^{*}\end{array}$ & $\begin{array}{l}\text { Incorporação de } \\
\text { novas faixas etárias, } \\
\text { não atingindo a } \\
\text { universalização (3)* }\end{array}$ & $\begin{array}{l}\text { Incorporação de } \\
\text { novas faixas etárias } \\
\text { e novas linhas de } \\
\text { cuidado (4)* }\end{array}$ \\
\hline Qualificação do CSB & $\begin{array}{l}\text { Formação não formal e } \\
\text { não instruído (1)* }\end{array}$ & $\begin{array}{l}\text { Formação não formal } \\
\text { e instruído (2)* }\end{array}$ & $\begin{array}{l}\text { Formação formal e } \\
\text { não instruído (3)* }\end{array}$ & $\begin{array}{l}\text { Formação formal } \\
\text { e instruído (4)* }\end{array}$ \\
\hline $\begin{array}{l}\text { Aumento dos } \\
\text { procedimentos da } \\
\text { atenção básica em } \\
\text { saúde bucal }\end{array}$ & $\begin{array}{l}\text { Não houve } \\
\text { aumento(1)* }\end{array}$ & $\begin{array}{l}\text { Houve aumento, mas } \\
\text { não soube quantificar } \\
(2)^{*}\end{array}$ & $\begin{array}{l}\text { Houve aumento e } \\
\text { soube quantificar } \\
(3)^{*}\end{array}$ & - \\
\hline $\begin{array}{l}\text { Referência } \\
\text { e contra-referência }\end{array}$ & $\begin{array}{l}\text { Não realiza referência } \\
(1)^{*}\end{array}$ & $\begin{array}{l}\text { M unicípio trabalha } \\
\text { não oficialmente } \\
\text { com rede de } \\
\text { referência }(2)^{*}\end{array}$ & $\begin{array}{l}\text { M unicípio conta } \\
\text { com rede de } \\
\text { referência } \\
\text { oficializada (3)* }\end{array}$ & - \\
\hline Relação CSB/ CDS & $\begin{array}{l}\text { Concentração de } \\
\text { responsabilidades e } \\
\text { informações }(1)^{*}\end{array}$ & $\begin{array}{l}\text { Descentralizada, sem } \\
\text { integração (2)* }\end{array}$ & $\begin{array}{l}\text { Descentralizada, } \\
\text { com integração (3)* }\end{array}$ & - \\
\hline $\begin{array}{l}\text { Conhecimento da } \\
\text { comunidade pelo CD }\end{array}$ & $\begin{array}{l}\text { Insatisfatório: os CDs } \\
\text { conhecem a área } \\
\text { adscrita, mas não } \\
\text { traçam perfil } \\
\text { epidemiológico da } \\
\text { população (1)* }\end{array}$ & $\begin{array}{l}\text { Satisfatório: } \\
\text { conhecimento da } \\
\text { área, da população } \\
\text { adscrita, } \\
\text { levantamento do } \\
\text { perfil epidemiológico } \\
\text { e eleição de } \\
\text { prioridades }(2) *\end{array}$ & - & - \\
\hline Relação CD/ equipe & $\begin{array}{l}\text { Isolada: o CD realiza o } \\
\text { seu planejamento e } \\
\text { executa as suas ações } \\
\text { sem a participação da } \\
\text { equipe }(1)^{*}\end{array}$ & $\begin{array}{l}\text { Integrada: o CD } \\
\text { participa das reuniões } \\
\text { de planejamento, } \\
\text { discussão de casos, } \\
\text { visitas domiciliares } \\
\text { com a equipe }(2)^{*}\end{array}$ & - & - \\
\hline $\begin{array}{l}\text { Clareza na descrição do } \\
\text { modelo de atenção em } \\
\text { Saúde Bucal pelo CSB }\end{array}$ & Não (1)* & $\operatorname{Sim}(2)^{*}$ & - & - \\
\hline
\end{tabular}

Fonte: Entrevista com os CSB dos municípios que compuseram a amostra.

* Pontuação atribuída à categoria. 
Assim, de acordo com essa escala, construiuse uma matriz de qualificação dos municípios, em que puderam ser classificados da seguinteforma: "estruturado", quando atingisse de 9 a 14 pontos; "semi-estruturado", quando atingisse de 15 a 20 pontos e "não estruturado", quando atingisse a pontuação entre 21 e 27.

0 presente estudo foi aprovado pelo Comitê de Ética e Pesquisa da ASCES (processo 145/05, aprovado em 16/02/2005).

\section{Resultadosediscussão}

Deacordo com a classificação metodológica adotada, três municípios enquadraram-se na categoria "estruturado" em relação ao modelo deatenção em saúde bucal implantado, cinco na cate goria "semi-estruturado" e um como "não estruturado", o que mostra que a maioria dos municípios analisados ( seis) ainda encontra dificuldades em estruturar a saúde bucal da sua população (Tabela 1).

Apesar da incorporação do CD à ESF, verifica-se ainda nessa tabela que a maior parte dos municípios da amostra apresentam poucos profissionais adequadamente qualificados no campo da saúde coletiva, pois dos nove municípios, quatro receberam o critério 3 (muitos CDs qualificados), três municípios receberam o critério 2 (poucos CDs qualificados) e 2 municípios receberam a pontuação 1 (nenhum CD qualificado), o que reproduz, além de uma prática pouco fundamentada nos princípios do SUS, um modelo ineficaz e de baixo impacto na saúde da popula- ção. N arvai ${ }^{9}$ afirma que os CDs continuam sendo formados de maneira desvinculada das reais necessidades do país, dissociados das características dos serviços nos quais irão atuar. Segundo Pedrosa e Tel es $^{10}$, as dificuldades de atuação desses profissionais na rede pública se devem à sua formação tecnicista e hospitalocêntrica, o que podeser confirmado através dos estudos de Funk et al. ${ }^{11}$, realizados no Rio Grande do Sul, onde $44,4 \%$ dos CD sentrevistados já fizeram uma pósgraduação e as áreas mais cursadas foram: endodontia, periodontia, prótese, odontopediatria e ortodontia.

A questão da baixa qualificação profissional também se estende aos CSB, pelo fato de apenas um município apresentar seu coordenador com qualificação estruturada (com pós-graduação na área de Saúde Pública e conscientedo modelo de atenção à saúde que coordena). Em três municípios, os coordenadores receberam a pontuação 1, ou seja, formação não formal e não instruído, conforme descrito na Tabela 1. Resultados semelhantes foram encontrados por Funk et al. ${ }^{11}$ os quais, estudando o perfil dosgestores da odontologia de dez cidades do RS, observaram que nove dos gestores entrevistados não tinham formação específica na área de Saúde Pública ou Saúde Bucal Coletiva. Encontraram também que a idéia de atenção básica da maioria dos entrevistados vincula-se à restauração, profilaxia, extração, urgência, e apenas um dos gestores referiu-se a questões de atenção integral e promo ção da saúde, concluindo que a capacitação dos gestores é uma das soluções para superar o modelo hegemônico de atenção à saúde.

Tabela 1. Matriz de qualificação dos municípios (M).

\begin{tabular}{lccccccccc}
\hline \multicolumn{1}{c}{ Questões avaliadas } & M 1 & M 2 & M 3 & M 4 & M 5 & M 6 & M 7 & M 8 & M 9 \\
\hline Qualificação dos CDs & 1 & 2 & 3 & 2 & 2 & 3 & 3 & 1 & 3 \\
Mudança no perfil da assistência & 3 & 2 & 4 & 2 & 4 & 3 & 4 & 2 & 1 \\
Procedimentos da atenção básica & 3 & 2 & 1 & 2 & 2 & 2 & 2 & 2 & 2 \\
Referência e contra-referência & 2 & 3 & 3 & 2 & 3 & 3 & 3 & 3 & 2 \\
Conhecimento da comunidade (CD) & 2 & 1 & 2 & 1 & 2 & 2 & 2 & 1 & 1 \\
Relação CD/Equipe & 2 & 2 & 2 & 2 & 2 & 2 & 2 & 2 & 2 \\
Modelo de atenção (CSB) & 1 & 1 & 2 & 1 & 1 & 1 & 2 & 1 & 1 \\
Qualificação do CSB & 2 & 2 & 2 & 1 & 2 & 1 & 4 & 1 & 3 \\
Relação CSB/CDS & 1 & 3 & 3 & 3 & 3 & 1 & 3 & 1 & 3 \\
TOTAL & 17 & 18 & 22 & 16 & 21 & 18 & 25 & 14 & 18 \\
Classificação final & SEM I & SEM I & EST & SEM I & EST & SEM I & EST & N E & SEM I \\
\hline
\end{tabular}

Nota: EST - estruturado; SEM I - semi-estruturado; NE - não estruturado. 
Dos nove CSB municipais entrevistados, oito não foram capazes de quantificar o aumento dos procedimentos da atenção básica, após incorporação do $C D$ na ESF, e sete não conseguiram explicitar com clareza o modelo deatenção em saúde bucal que coordenam (Tabela 1). Esses resultados apontam como um indicativo de que alguns profissionais não procuram planejar, avaliar econtrolar suas atividades en quanto CSB, além de não compreenderem o modelo de aten ção e a filosofia da ESF, o que resulta em práticas pouco efetivas e não condizentes às reais necessidades de saúde da população.

Em relação à política nacional de saúde bucal, percebe-se, com clareza, a preocupação com a reorganização da atenção à saúde bucal, adotando-se como eixo de reorientação do modelo o conceito do cuidado, compreendido como capaz de promover boa qualidade de vida e de intervir nos fatores que a colocam em risco ${ }^{12}$.

Enquanto a relação $C D /$ Equipe apresentouse estruturada para todos os municípios, o mesmo não ocorreu com a relação CSB/CD, em que três municípios apresentaram situação não estruturada, o que demonstra que, em alguns municípios, ainda há concentração de responsabilidades e informações, e dificuldade para se trabaIhar em equipe, impedindo a efetivação de um modelo condizente com a reorientação das práticas em saúde. Pedrosa e Teles ${ }^{10}$ observaram em um estudo sobre o PSF em Teresina, Piauí, que o relacionamento interno da equipe analisada re velou a inexistência de responsabilidade coletiva pelos resultados do trabalho, levando à descontinuidade entre as ações específicas de cada profissional, observando-se desarticulação entre ações curativas, educativas e administrativas, bem como um baixo grau de interação entre os membros da equipe.

Também chama a atenção a falta de uma rede dereferência econtra-referência oficializada para a saúde bucal, verificada em quatro dos municípios, o que compromete a qual idade da assistência prestada e não assegura à população assistência nos demais níveis de complexidade.

Como a ESF caracteriza o primeiro contato da população com o serviço de saúde municipal, deve-se garantir resolutividade dos casos, assegurando-sereferência econtra-referênciacom os diferentes níveis do SUS, de modo a "reverter" a lógica do modelo vigente" ${ }^{\prime \prime}$.

\section{Conclusão}

Dessa forma, percebe-se que a maioria dos municípios pesquisados (seis) apresenta dificuldades na estruturação da saúde bucal à luz da Estratégia de Saúde da Família. 0 fato de o perfil profissional estar voltado para a clínica, influência de uma formação flexineriana, e também a expectativa da comunidade, habituada ao atendimento curativo do profissional, refletem um atendimento meramente clínico e pontual.

A ausência de uma pós-graduação baseada nas premissas do SU $S$, comum à maioria dos CD s e CSB entrevistados, aliada à formação acadêmica curativa supracitada, demonstram a falta de preparo do profissional para uma atuação mais adequada ao PSF. Dessa forma, as questões relacionadas à incorporação de novas práticas assistenciais, bem como 0 entendimento desses atores na rede de referência e contra- referência, estão comprometidas.

A motivação desses profissionais deveser uma prioridade; 0 investimento e estímulo à educação permanente e um monitoramento e avaliação das ações por eles desenvolvidas podem marcar o início do redirecionamento do modelo assistencial em Saúde Bucal.

\section{Colaboradores}

PJL M artelli coordenou todas as fases da pesquisa, desde a coleta de dados à aprovação da versão para publicação; APS Cabral, CLSV M acedo eSF Silva participaram da concepção da pesquisa, coleta de dados, metodologia, análise, correções e aprovação da versão a ser publicada; FC Pimentel e IS M onteiro participaram da análise, revisão da literatura, discussão dos resultados, elaboração dos quadros e redação do artigo. 


\section{Referências}

1. Martelli PJLM . Análise da atenção em saúde bucal no nível municipal: caracterização do modelo assistencial [dissertação]. Recife (PE): Departamento de Saúde Coletiva, Centro de Pesquisas Aggeu M agalhães, Fundação Oswaldo Cruz; 2001.

2. Zanetti CHG. Por um caminho sustentável para universalização da atenção básica. Departamento de Odontologia, Unb. Brasília, 2000. [acessado 2006 mai 19]. Disponível em: http://www.saudebucal coletiva.unb.br/oficina/estado/coletivo_amplo/ psf_caminho.htm

3. Oliveira AGRC, Arcieri RM, Unfer B, Costa ICC, M oraes E, Saliba NA. Modelos assistenciais em saúde bucal no Brasil: tendências e perspectivas. A ção Coletiva 1999; 2(1):9-14.

4. Silva SF. A saúde bucal dentro da estratégia de saúde da família no estado de Pernambuco [monografia]. Recife (PE): Centro de Pesquisas Aggeu Magalhães, Fundação Oswaldo Cruz; 2006.

5. Albuquerque RRS. Contribuições para a discussão sobre a formação do odontólogo a partir da inserção da saúde bucal no PSF [dissertação]. Rio de Janeiro (RJ): Escola N acional de Saúde Pública Sérgio ArouCa; 2004.

6. Trad LAB, Bastos ACS. 0 impacto sócio-cultural do Programa de Saúde da Família (PSF): uma proposta de avaliação. Cad Saúde Pública 1998; 14:429-435.

7. Feuerwerker LCM. Educação dos profissionais de Saúde hoje. Rev. ABENO 2003; 3(1):24-27.
8. O movimento de mudança da formação de profissionais de saúde no Brasil. Divulg. saúde debate 2000; 22:4-7.

9. Narvai PC. Odontologia e saúde bucal coletiva. São Paulo: Hucitec; 1994.

10. Pedrosa JTS, Teles JBM. Consenso e diferenças em equipes do Programa Saúde da Família. Rev. Saúde Pública 2001; 35:303-311.

11. Funk PP, Flôres M MDZ, Garbin CA, Hartmann MSM, M endonça JL. Perfil do profissional formado pela faculdade de Odontologia da Universidade de Passo Fundo/RS: da formação à realidade profissional. Passo Fundo 2004; 9(2):105-109.

12. Brasil. Ministério da Saúde. Secretaria de Atenção à Saúde. Departamento de Atenção Básica. Coordenação Nacional de Saúde Bucal. Diretrizes da Política Nacional de Saúde [periódico na internet] Brasília, 2004 [acessado 2006 jul 8]. Disponível em: http://portal.saude.gov.br/portal/arquivos/pdf/ politica_nacional_brasil_sorridente.pdf

13. Veras $C \bar{M} M$, Rodrigues R $\bar{M}$, Carvalho ALB. O Saúde da Família na Paraíba: do processo de implantação aos resultados. Divulg. saúde debate 2000; 20: 27-34.

Artigo apresentado em 27/09/2006

Aprovado em 30/01/2007

Versão final apresentada em 30/01/2007 